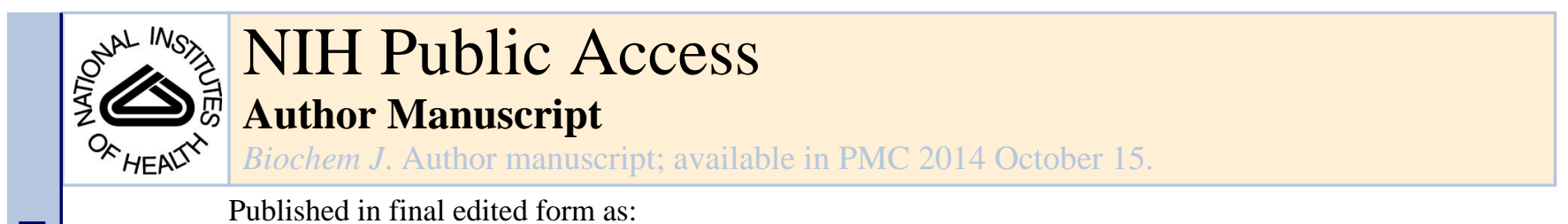

Published in final edited form as:

Biochem J. 2012 October 1; 447(1): 159-166. doi:10.1042/BJ20120938.

\title{
RSK phosphorylates SOS1 creating 14-3-3 docking sites and negatively regulating MAPK activation
}

\author{
Madhurima Saha*, Audrey Carriere ${ }^{\dagger, \ddagger, 1}$, Mujeeburahiman Cheerathodi ${ }^{\star}$, Xiaocui Zhang $^{\dagger}$, \\ Geneviève Lavoie $^{\dagger}$, John Rush ${ }^{\S}$, Philippe P. Roux ${ }^{\dagger, \ddagger, 2}$, and Bryan A. Ballif ${ }^{\star}, 2$ \\ *Department of Biology, University of Vermont, Burlington, VT, 05405, U.S.A. \\ †Institute for Research in Immunology and Cancer (IRIC), Montréal, Québec, Canada H3C 3J7 \\ FDepartment of Pathology and Cell Biology, Faculty of Medicine, Université de Montréal, \\ Montréal, Québec, Canada H3C 3J7 \\ §Cell Signaling Technology, Inc., 3 Trask Lane, Danvers, MA, 01923, U.S.A.
}

\author{
Keywords \\ RSK; SOS; 14-3-3; negative feedback; Phosphorylation; Signal Transduction
}

\section{INTRODUCTION}

Cells respond to an array of biological and environmental stimuli which trigger intracellular signaling pathways governing a diversity of cellular states. Differences in the magnitude and duration of signaling can lead to very different biological outcomes. This has been studied by examining cellular commitment to proliferation as a function of strength and duration of Ras-MAPK signaling in mammalian cells [1]. Intriguingly, while some cells proliferate due to increased Ras-MAPK signaling, others have varied responses including differentiation, senescence, survival and death [2-5]. Thus, genetic and biochemical context can dramatically alter the cellular interpretation of a given signal.

Furthermore, while activating mutations of Ras-MAPK signaling can be found in greater than $30 \%$ of all human tumors, and greater than $60 \%$ in specific tumor types such as in pancreatic cancers [5-8], hyperactive Ras-MAPK signaling is responsible for several human developmental disorders which display defects which can't be defined simply or exclusively as over-proliferation disorders. These so-called Rasopathies include Neurofibromatosis 1,

\footnotetext{
${ }^{2}$ To whom correspondence should be addressed: Dr. Bryan A. Ballif, Department of Biology, University of Vermont, 109 Carrigan Drive, Burlington, VT, 05405, USA; Tel.: (802) 656-1389; Fax: (802) 656-2914; bballif@uvm.edu. Dr. Philippe P. Roux, IRIC Université de Montréal, P.O. Box 6128, Station "Centre-Ville", Montréal, Québec, Canada H3C 3J7; Tel.: (514) 343-6399; Fax: (514) 343-5839; philippe.roux@umontreal.ca.

${ }^{1}$ Current address: Centre National de la Recherche Scientifique (CNRS), UMR 5241 UPS EFS U1031 Inserm STROMALab, BP 84 225, F-31 432 Toulouse, France.

AUTHOR CONTRIBUTIONS

Madhurima Saha and Audrey Carriere conducted the majority of the experiments with additional experiments performed by Mujeeburahiman Cheerathodi, Xiaocui Zhang, Geneviève Lavoie, Philippe Roux and Bryan Ballif. John Rush helped design and provided the stable isotope-containing reference peptides. Bryan Ballif wrote the paper with the assistance of Philippe Roux. Bryan Ballif and Philippe Roux were the principals directing and designing experiments and interpreting the data.
} 
Noonan syndrome, LEOPARD (multiple lentigines, electrocardiographic conduction abnormalities, ocular hypertelorism, pulmonary stenoisis, abnormal genitalia, retardation of growth and sensorineural deafness) syndrome, Costello syndrome and CFC (cardio-faciocutaneous) syndrome [8-10]. Together, these disorders display a striking variety of cellular phenotypes and clinical manifestations underscoring not only the importance of developing therapeutic approaches to control Ras-MAPK signaling but obviating the need for evolved cellular mechanisms of developmental and homeostatic pathway control.

A number of molecular mechanisms have been uncovered that negatively regulate RasMAPK signaling in vivo. These include dephosphorylation of activating phosphorylation events, inhibitory binding of 14-3-3 to pathway components Raf [11, 12] and KSR [13, 14], and negative feedback loops. The known negative feedback loops are dependent on the activation of the canonical MAP kinases ERK1/2 (extracellular regulated kinases 1 and 2) which desensitize Raf-1 by direct phosphorylation [11]; which phosphorylate the RasGEF Son of Sevenless 1 (SOS1) leading to SOS1 dissociation from Grb2 (the adaptor protein which links SOS1 to tyrosine phosphorylated growth factor receptors and scaffolds [15]); and which transcriptionally regulate dual-specificity phosphatases [16]. In recent years, an additional negative feedback mechanism of the Ras-MAPK pathway was experimentally observed when pharmaceutical and genetic disruption of the MAPK-activated $90 \mathrm{kDa}$ Ribosomal S6 Kinase (RSK) family led to increased or sustained ERK1/2 activity [17-19]. Based on the evidence that RSK could phosphorylate SOS1 in vitro [20], it has been assumed that RSK mediates negative feedback in Ras-MAPK signaling via modulation of SOS1. However, the demonstration of RSK phosphorylation of SOS1 in cells and its putative negative regulatory effect on MAPK activation is still lacking.

Here we establish that RSK phosphorylates SOS1 in vivo at Ser1134 and Ser1161. Furthermore, we show that RSK-dependent phosphorylation of these residues creates 14-3-3 binding sites on SOS1. Finally, expression of an unphosphorylatable form of SOS1 modestly increases ERK1/2 activation and duration in response to epidermal growth factor (EGF) stimulation. Together these data indicate that RSK phosphorylation of SOS1 is one mechanism whereby RSK provides negative feedback control in Ras-MAPK signaling. However, these data also argue for additional mechanisms of RSK-dependent negative feedback to MAPK activation that remain to be identified.

\section{Experimental}

\section{Plasmids and Site-Directed Mutagenesis}

The following plasmid constructs were described previously: pGEX-2TK-14-3-3 $\beta$ (human), pGEX-4T-14-3-3 $\varepsilon$ (rat) wild-type and K49E [21] (M. Yaffe), pCGN-SOS1-HA [15] (D. Bar-Sagi), pCMV6-Myristoylated-Rsk1 (avian) [22], pKH3-HA-RSK2 (mouse) [23], FlagMEK1-DD [24]. For the expression of GST-14-3-3e variants in mammalian cells we PCR amplified 14-3-3 $\varepsilon$ wild-type and K49E in pGEX-4T inserting a 5' BamHI site and a 3' NotI site which was then ligated in-frame with GST in pEBG (B. Mayer). The generation of the Ser1134Ala and Ser1161Ala mutants was done using the QuikChange strategy (Stratagene) and mutants were verified by DNA sequencing at the Vermont Cancer Center's DNA Analysis Facility. 


\section{GST-14-3-3 $\varepsilon$ Fusion Proteins}

$50 \mathrm{ml}$ overnight cultures of BL21 E. Coli transformed with pGEX-4T-14-3-3 $\beta$, $\varepsilon$ wild-type or K49E were diluted into $500 \mathrm{ml}$ and cultured for two hours followed by induction with $1 \mathrm{mM}$ Isopropyl $\beta$-D-1-thiogalactopyranoside (IPTG) for six hours. Cells were pelleted and resuspended in $10 \mathrm{ml}$ of phosphate-buffered saline (PBS), $0.1 \mathrm{M}$ EDTA, $5 \mathrm{mg} / \mathrm{ml}$ pepstatin A, $10 \mathrm{mg} / \mathrm{ml}$ leupeptin and $1 \mathrm{mM}$ phenylmethylsulfonyl fluoride (PMSF). The suspensions were sonicated on ice using a probe sonicator eight times for thirty seconds with 30 second delays between blasts. $1 \mathrm{ml}$ of Triton X-100 was then added and the sonicates were centrifuged at 13,000 X g for 30 minutes. The supernatants were incubated with $300 \mu \mathrm{l}$ of a washed 50\% slurry of glutathione agarose (G Biosciences, Maryland Heights, MO). The beads were washed four times with the bacterial lysis buffer with $1 \%$ Triton X-100 and then three times with mammalian cell lysis buffer (see below). All manipulations were performed at $4{ }^{\circ} \mathrm{C}$.

\section{Cell Culture, Transfections, Pulldown Assays, Immunoprecipitation, Immunoblotting, Densitometry, Antibodies and RNA Interference}

E1A-transformed human embryonic kidney 293 (HEK 293), COS7, NIH3T3 and mouse embryonic fibroblasts (MEFs) immortalized using the "3T3" protocol were maintained in DMEM (Mediatech, Manassas, VA) supplemented with 10\% fetal bovine serum (Hyclone, Logan, UT), 50 units $/ \mathrm{mL}$ penicillin, and $50 \mu \mathrm{g} / \mathrm{mL}$ streptomycin. For stimulations cells were starved of serum for 16-18 hours prior to treatments. Pharmacological inhibitors and stimulants were from the following sources (with final concentrations indicated): BI-D1870 (10 $\mu \mathrm{M}$; Biomol, Plymouth Meeting, PA), SL0101 (50 $\mu \mathrm{M}$; Toronto Research Chemicals),

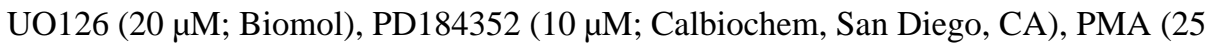
$\mathrm{ng} / \mathrm{ml}$; Biomol and Calbiochem) EGF ( $25 \mathrm{ng} / \mathrm{ml}$; Invitrogen, Carlsbad, CA, and Cell signaling Technology, Beverly, MA). Transfections were done by calcium phosphate precipitation. For pulldowns and the endogenous co-immunoprecipitations, cells were lysed in $25 \mathrm{mM}$ Tris, pH 7.4, $137 \mathrm{mM} \mathrm{NaCl}, 1 \%$ Igepal, $10 \%$ glycerol, $25 \mathrm{mM} \mathrm{NaF}, 10 \mathrm{mM}$ $\mathrm{Na}_{4} \mathrm{P}_{2} \mathrm{O}_{7}, 1 \mathrm{mM} \mathrm{Na} \mathrm{VO}_{4}, 5 \mathrm{mg} / \mathrm{ml}$ pepstatin A, $10 \mathrm{mg} / \mathrm{ml}$ leupeptin and $1 \mathrm{mM}$ PMSF. Clarified lysates (containing $~ 500 \mu \mathrm{g}$ protein) were incubated with $30 \mu \mathrm{l}$ of a $30 \%$ slurry of GST-14-3-3 $\varepsilon$ wild-type or K49E. After incubating two hours to overnight the beads were washed four times with lysis buffer prior to SDS-PAGE and immunoblotting. For anti-HA immunoprecipitations cells were lysed in $0.5 \%$ NP40 (or Igepal), $0.1 \%$ Brij-35, $0.1 \%$

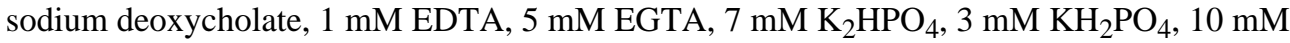
$\mathrm{MgCl}_{2}, 50 \mathrm{mM} \beta$-glycerolphosphate, $1 \mathrm{mM} \mathrm{Na} \mathrm{VO}_{4}, 5 \mu \mathrm{g} / \mathrm{ml}$ pepstatin $\mathrm{A}, 10 \mu \mathrm{g} / \mathrm{ml}$ leupeptin and $1 \mathrm{mM}$ PMSF. Immunoblotting was performed using a submersible transfer apparatus, and nitrocellulose membranes. Blocking was performed in 5\% milk/TBST (0.05\% Tween-20, $8 \mathrm{mM}$ Tris-Base, $25 \mathrm{mM}$ Tris-HCL, $154 \mathrm{~m} \mathrm{M} \mathrm{NaCl})$. Primary antibodies were incubated with the membranes in $1.5 \%$ BSA in TBST and washes were done with TBST. Secondary antibodies conjugated to horse radish peroxidise were from Chemicon/ Millipore (Billerica, MA) and visualization was done using enhanced chemiluminescence and exposure to X-ray film. Densitometry analysis was conducted using identical areas for each lane of a given blot using inverted histograms in Adobe Photoshop CS2. After subtracting the background, phospho-ERK1/2 levels were normalized to the levels of total 
ERK1/2 and HA-SOS1 levels. Commercial primary antibodies were from the following sources: anti-Rsk1 used in Figures 3E and 4C (Santa Cruz Biotechnology, Santa Cruz, CA), anti-Rsk1 used in Figures 1A-B and 2A (Cell Signaling Technology), anti-Rsk2 used in Figure 1B (Cell Signaling Technology, Beverly, MA), anti-Ser380 phospho-Rsk (Cell Signaling Technology), anti-GST (Upstate Biotechnology/Millipore, Billerica, MA), antiHA (Covance, Princeton, New Jersey), anti-Thr202/Tyr204 phospho-Erk1/2 (Cell Signaling Technology), anti-SOS1 (Upstate Biotechnology/Millipore), and anti-phospho-Akt Substrate (anti-RXXpS, Cell Signaling Technology). Anti-avian Rsk1 and anti-Erk1/2 were gifts from J. Blenis and were described previously [25]. Small interfering RNA (siRNA) against RSK1 (SI02223067), RSK2 (SI00288190) and the negative control siRNA (1027280) were obtained from QIAGEN (Valencia, CA). 1-2 $\mu \mathrm{g}$ (50 nmoles) of siRNA were transfected per $6 \mathrm{~cm}$ dish using calcium phosphate precipitation as described [26].

\section{Quantitative Mass Spectrometry and Data Analysis}

Immunoprecipitated HA-SOS1 from transfected cells starved of serum and then untreated or stimulated with either EGF or PMA was subjected to SDS-PAGE and staining with Coomassie blue. Bands were digested in-gel with trypsin, and tryptic peptides were extracted and prepared as described [27]. Dried peptides were resuspended in $8.5 \mu \mathrm{l}$ of $2.5 \%$ acetonitrile and $2.5 \%$ formic acid containing $100 \mathrm{fmol} / \mu \mathrm{l}$ of each stable isotope-containing, absolute quantification (AQUA) [28] peptide standard prior to liquid chromatography tandem mass spectrometry analysis (LC-MS/MS) in a linear ion trap-orbitrap hybrid mass spectrometer (Thermo Electron, San Jose, CA) set up and run as described [29]. Two $4 \mu \mathrm{l}$ technical replicates were analyzed and quantified using monoisotopic peak heights as described [30, 31]. For quantification of fold changes, the abundance of each peptide was first determined by dividing the ratio of the labelled (heavy) peptide to the native (light) peptide and then normalizing this ratio by dividing it by the heavy/light ratio of the reference peptide Phe648-Arg660 found in the same sample. The fold changes were then determined by dividing the ratios obtained from stimulated samples by the ratios obtained in the unstimulated states. Technical replicates were averaged and the standard deviations were calculated. Supplementary Figure S1 shows representative MS/MS spectra for each heavy reference peptide and the corresponding identified native peptides. Stable isotope-containing heavy "AQUA" peptides were synthesized at Cell Signaling Technology.

\section{Results}

\section{The Ras-MAPK pathway promotes SOS1 phosphorylation at basophilic sequences via RSK}

RSK, like many of its basophilic kinase relatives, shows a preferred minimal substrate target motif, Arg-X-X-Ser [32-34], where $\mathrm{X}$ is any amino acid and Ser is the serine targeted for phosphorylation. Supplementary Figure S2 lists a selection of 18 characterized RSK substrates $[33,34]$ and the amino acid sequences surrounding the sites of phosphorylation. Also shown are two strong potential RSK target sites (Ser1134 and Ser1161) in the carboxyl-terminus of SOS1 (not found in SOS2) which exhibit high conservation among vertebrate SOS1 orthologs. Given SOS1 has putative RSK phosphorylation sites we asked if EGF or the phorbol ester PMA (phorbol 12-myristate 13-acetate), both known in vivo 
activators of RSK [34, 35], would induce phosphorylation of SOS1 in a minimal RSK consensus motif. We made use of a phospho-specific motif antibody that recognizes the phosphorylated minimal RSK consensus motif (RXXpS), where $\mathrm{pS}$ is phosphoserine and X is any amino acid) [26, 35], and we observed increased SOS1 phosphorylation following both EGF and PMA stimulation. This phosphorylation was blocked when cells were pretreated with inhibitors of MAPK-Kinase (MEK) activation (UO126 and PD184352) or RSK (BI-D1870) (Fig. 1A). Furthermore, overexpression of a constitutively active allele of MEK1 (MEK-DD)[24] induced SOS1 phosphorylation as recognized by the anti-RXXpS motif antibody (Fig. 2B). This effect was eliminated by RNAi-dependent silencing of RSK1 and RSK2, with RSK2 silencing having the more profound effect (Fig. 1B). Together these data provided strong evidence for the presence of at least one in vivo RSK phosphorylation site on SOS1.

\section{RSK phosphorylates SOS1 at serines 1134 and 1161}

Earlier work described RSK phosphorylation of SOS1 in vitro using purified RSK, ${ }^{32} \mathrm{P}-\gamma$ ATP and a GST-carboxyl-terminal (Ser1132-Ser1343) SOS1 [20]. Although not definitive, phosphopeptide mapping best placed a single phosphorylation site at Ser1161 [20]. Of note, Arg1131 was not present in the GST-carboxyl-terminal SOS1 fusion and would therefore have truncated the RSK target motif surrounding Ser1134. To definitively monitor PMAand EGF-induced changes in SOS1 Ser1134 and Ser1161 phosphorylation, we took a quantitative mass spectrometry (MS) approach using stable isotope-containing "heavy" reference peptides [28]. The advantage of this quantitative MS approach is that a given native peptide can be quantified directly relative to a known amount of the internal reference peptide based on the fact that the ionization efficiency and chromatographic behavior of the two peptides are identical. Furthermore, the collision-induced dissociation (CID) tandem mass spectra can provide unambiguous identification and quantification of site-specific phosphorylation (see below and Supplementary Fig. S1). To monitor site-specific changes in Ser1134 and Ser1161 phosphorylation we immunoprecipitated HA-SOS1 from cells stimulated with either EGF or PMA. Ten percent of each immunoprecipitation was subjected to western blotting using the RXXpS motif antibody and an increase in phosphorylation of the putative RSK target motif was confirmed (Fig. 2A). The rest of the immune complex was subjected to SDS-PAGE and staining with Coomassie blue (Fig. 2B). The bands were digested in-gel with trypsin and extracted peptides were resuspended in a solution containing a fixed amount of each stable isotope-containing peptide standard prior to liquid chromatography tandem mass spectrometry analysis (LC-MS/MS) in a linear ion trap-orbitrap hybrid mass spectrometer. The heavy reference peptides included peptides harboring Ser1134 and Ser1161 in their phosphorylated and unphosphorylated states. Given that ERK1/2 phosphorylate the proline-directed Ser1167 in the same tryptic peptide as Ser1161, we monitored the doubly phosphorylated phospho-Ser1161, phospho-Ser1167containing peptide. To normalize SOS1 protein levels across samples, an additional heavy peptide (Phe648-Arg660) was monitored that was not predicted to become modified following stimulation. Tandem mass spectra for all native and reference peptides are provided in Supplementary Figure S1. Exemplifying the raw data, Fig. 2C displays the MS1 spectra for the heavy and native unphosphorylated and phosphorylated Ser1134-containing peptides as well as peptide Phe648-Arg660 of SOS1 immuno-purified from starved, PMA- 
stimulated and EGF-stimulated conditions. An increase in the relative abundance of the phospho-Ser1134-containing peptide was observed following PMA and EGF stimulation with an expected corresponding decrease in the unphosphorylated Ser1134-containing peptide. After technical replicates were analyzed the average fold changes were plotted (Fig. 2D). The quantitative MS data are in good agreement with the anti-RXXpS data shown in Figure 2A and definitively show PMA- and EGF-induced phosphorylation of Ser1134, Ser 1161 and Ser1167. To determine if Ser1134 and Ser1161 were the major SOS1 sites of antiRXXpS immunoreactivity, we mutated these residues singly or in combination to generate non-phosphorylatable alanine mutants. These HA-tagged constructs were expressed in cells as well as wild-type HA-SOS1. Cells were starved of serum and either left untreated or stimulated with PMA or EGF. Anti-HA immune complexes and immunoblotting with the anti-RXXpS antibody showed that Ser1134 is the major RXXpS site on SOS1 followed by Ser1164 in agreement with the MS data. The double mutant showed no appreciable stimulation-induced phosphorylation (Fig. 2E).

\section{Phosphorylation of SOS1 at serines 1134 and 1161 creates 14-3-3 binding sites}

The minimal target motif (RXXS) as visualized by a frequency plot [36] of RSK substrates overlaps with the preferred mode 1 binding motif (RSXpSXP, Fig. 3A) of the 14-3-3 family of phosphoprotein regulators [21,37]. As SOS1 Ser1134 and Ser1161 each show conserved aspects of the canonical mode 1 14-3-3 binding motif (Fig. 3A), and given that 14-3-3 has been shown to negatively regulate Ras-MAPK signaling by binding to both Raf and KSR, we asked if SOS1 might also bind to 14-3-3 in a phospho-dependent manner. We expressed HA-SOS1 in HEK 293 cells and lysed the cells while they were growing in complete media. Extracts were subjected to a GST-14-3-3 pulldown assay using either wild-type 14-3-3ع or a K49E mutant that shows dramatically reduced binding to phosphorylated substrates [38]. Consistent with a phospho-dependent interaction, HA-SOS1 bound to wild-type 14-3-3e but showed little to no binding to the K49E mutant (Fig. 3B). Similar results were observed using 14-3-3 $\beta$ (Fig. 3C) and when using transfected HA-SOS1 with transfected GST-14-3-3 (Fig. 3D). We also observed PMA-induced binding of endogenous SOS1 with GST-14-3-3 in a pulldown assay from three immortalized cell lines (Fig. 3C, 3E).

\section{RSK regulates 14-3-3 binding to SOS1 in response to Ras-MAPK activation}

To determine if RSK is the kinase responsible for the interaction of 14-3-3 with SOS1, we examined the interaction from three angles. First, we asked whether the PMA-induced binding of SOS1 to 14-3-3 would be blocked by pharmacological inhibition of MEK-MAPK signaling and found that it was the case (Fig. 4A). Second, we expressed a constitutively active allele of RSK1 [22] and found that 14-3-3 binding to SOS1 was dramatically induced in serum-starved cells (Fig. 4B). Finally, we knocked down RSK expression using RNA interference and found this prevented the PMA- and EGF-induced interaction of SOS1 with 14-3-3 (Fig. 4C). We next examined the functional consequences of a SOS1 allele that could not be phosphorylated at the RSK phosphorylation sites. The SOS1 Ser1134Ala,

Ser1161 Ala double mutant displayed no detectable binding to 14-3-3 and while the two sites each contributed to the binding to 14-3-3, phosphorylation at Ser1134 was most important (Fig. 4D). Together, these data demonstrate that RSK regulates14-3-3 binding to SOS1 by promoting phosphorylation of Ser1134 and Ser1161. 


\section{SOS1 phosphorylation by RSK inhibits EGF-induced MAPK activation}

We next examined a time course of EGF-dependent ERK1/2 activation in cells overexpressing either wild-type SOS1 or the SOS1 Ser1134Ala, Ser1161Ala double mutant. Consistent with phosphorylation of these sites contributing to negative regulation of RasMAPK signaling, we observed an increase in the magnitude and duration of EGF-dependent ERK1/2 activation (Fig. 5A-B). These data support the notion that RSK is a negative regulator of Ras-MAPK activity, and is consistent with previous literature, we found that pharmacological disruption of RSK using two different inhibitors (BI-D1870 and SL0101) resulted in increased ERK1/2 phosphorylation in response to EGF stimulation in HEK 293 cells (Fig. 1A) and MEFs (Fig. 5C). The effect of the RSK inhibitors appeared stronger compared to the effect of overexpressing the double phosphorylation site SOS1 mutant, but this could be due to several factors including contributions of endogenous SOS1 in the overexpression experiments, compensatory effects during the transfection time compared to acute effects during drug treatments, or that SOS1 is not the only target of RSK-dependent negative feedback on Ras-MAPK signaling. Together, our results demonstrate that RSK phosphorylates SOS1 on residues that create 14-3-3 binding sites and which play a role in the negative regulation of the Ras-MAPK pathway.

\section{DISCUSSION}

The importance of understanding the various molecular mechanisms negatively regulating Ras-MAPK signaling is profound given the prevalence of its hyperactivation in human tumors as well as in rare but devastating, developmental disorders. Notwithstanding the strong pharmacological and genetic evidence for a negative feedback role for RSK in RasMAPK signaling, little progress toward elucidating the relevant molecular mechanisms has been made. Here we identify RSK phosphorylation sites on SOS1 that negatively regulate EGF-dependent ERK1/2 activation, potentially as they create 14-3-3 binding sites that could interfere with molecular interactions of Ras pathway components. 14-3-3 has already been shown to interfere with other components of Ras-MAPK signaling, including Akt phosphorylated Raf $[11,12,39]$, and the MAPK signaling cassette scaffold, KSR phosphorylated by C-TAK1 [13]. We propose a two stage negative feedback loop toward SOS1 in Ras-MAPK signaling with the first stage at the level of ERK1/2 and the second stage at the level of RSK (Fig. 6). ERK1/2 have long been known to phosphorylate SOS1 at four critical residues reducing the binding of SOS1 to GRB2 [15], the effect of which may be to diffuse signaling away from the receptor complex even if SOS1's membrane binding domains retain SOS1 near lipid products at the membrane. In stage two of the negative feedback loop RSK phosphorylation of SOS1 induces 14-3-3 binding which may prevent the interaction of SOS1 with Ras by directly blocking the interaction or by inducing conformational changes that reduce the interaction of SOS1 with Ras, or with the membrane itself (Fig. 6). Evidence suggesting that the negative effect of RSK is functioning upstream of Raf can be found in Figure 1A, where the RSK-specific inhibitor BI-D1870 leads to a dramatic upregulation of ERK1/2 phosphorylation following EGF stimulation but this is not observed following PMA stimulation. This is consistent with the mechanism of action of PMA at the level of Raf [40]. 
A large proportion of human cancers are characterized by hyperactivation of the MAPK pathway, whereby deregulated ERK1/2 helps drive unrestricted cell growth and proliferation. RSK was confirmed to be constitutively active in several cancers [41], such as melanoma [42], suggesting that it regulates substrates that contribute to tumourigenesis. Based on our findings and that of others $[17,18]$, RSK negatively regulates MAPK activation in response to growth factors. Thus, in addition to its growth-related functions, RSK may also limit MAPK signaling in cells with perturbed Ras regulation, such as in neurofibromatosis-1 (NF-1) [43]. The biological impact of RSK activation is therefore the result of its dual roles downstream of the MAPK pathway, and it is conceivable that hyperactivation of RSK may in some cases be detrimental to MAPK-dependent cell proliferation. Very few studies have reported increased tumor-derived RSK expression. Rather, accumulating evidence support the idea that at least some RSK isoforms are downregulated in certain cancers (in particular RSK3 and RSK4 [44, 45]). While our data indicate a requirement for RSK1 and RSK2 (with RSK2 playing a more important role) in Ser1134 and Ser1161 phosphorylation of SOS1 in response to growth factor stimulation, further investigation will be necessary to determine the extent RSK3 or RSK4 participate in the regulation of SOS1 in other cellular contexts. However, significant disruption of negative feedback is observed in muscle and neuronal cells of RSK2-deficient animals [18, 19] suggesting RSK2 plays a primary role in the negative feedback mechanism.

Recently, canonical mammalian Ras-MAPK signaling has been characterized as a system with significant robustness to change, given its graded, rather than switch-like, signal amplification [46-48]. The robustness of the system is dependent on intact negative feedback loops and is characteristic of negative feedback amplification systems in engineering that facilitate the buffering or smoothening of a given output even when inputs fluctuate [16, 46, 47]. While ERK phosphorylation of Raf was observed by others to provide strong negative feedback in serum- and EGF-dependent signaling [16, 47], genetic disruption of RSK2 alone leads to significant loss of negative feedback following insulin stimulation in skeletal muscle and glutamate signaling in neurons $[18,19]$.

To understand the topology of any signaling system, it is important to delineate the strength and multiplicity of negative feedback mechanisms acting within it. This is particularly true if imposition of targeted pharmacological control is desired as is the case with hyperactive Ras-MAPK signaling. For example, if RSK inhibitors were to be used as anti-cancer therapy then they would likely be more effective when tumors are driven by activating mutations in RAF since RSK-dependent negative feedback appears to act upstream of RAF, at least in part on SOS1. Thus, the RSK-dependent negative feedback mechanisms, in part described herein, highlight the need for critical evaluation of both the positive and negative contributions initiated by RSK isoforms in Ras-MAPK signaling in both normal and aberrant biology.

\section{Supplementary Material}

Refer to Web version on PubMed Central for supplementary material. 


\section{Acknowledgments}

We acknowledge M. Yaffe, D. Bar-Sagi, B. Mayer and J. Blenis for reagents; J. Reynolds and J. Knott for AQUA peptide preparation; and the Vermont Cancer Center DNA Analysis Facility for DNA sequencing.

FUNDING

This work was supported by the Vermont Genetics Network through NIH grant P20 RR16462 from the INBRE Program of the NIMGS (B.A.B. and M.S.), and by grants 700878 and DF121153 from the Canadian Cancer Society Research Institute and the Cancer Research Society, respectively (P.P.R.). P.P. Roux holds a Canada Research Chair in Signal Transduction and Proteomics. A. Carrière is recipient of a fellowship from the Cole Foundation, and Xiaocui Zhang was awarded a doctoral studentship from the China Scholarship Council (CSC). The University of Vermont neuroscience molecular core provided film developing equipment and is funded by NIH COBRE grant 5 P20 RR016435.

\section{Abbreviations}

MAPK

ERK

Ras

SOS1

RSK

LEOPARD

CFC

GEF

AQUA

CID

HEK

PMA

EGF

KSR

C-TAK

RAF

MEK
Mitogen Activated Protein Kinase

Extracellular Signal-Regulated Kinase

rat sarcoma

Son of Sevenless 1

Ribosomal S6 Kinase

multiple lentigines, electrocardiographic conduction abnormalities, ocular hypertelorism, pulmonary stenoisis, abnormal genitalia, retardation of growth and sensorineural deafness

cardio-facio-cutaneous

Guanine Nucleotide Exchange Factor

absolute quantification

collision-induced dissociation

Human Embryonic Kidney

phorbol-12-myristate-13-acetate

Epidermal Growth Factor

Kinase Suppressor of Ras

Cdc twenty-five $\mathrm{C}$ associated protein kinase

Mitogen Activated Protein Kinase Kinase Kinase (MAPKKK)

Mitogen Activated Protein Kinase Kinase

\section{References}

1. Murphy LO, MacKeigan JP, Blenis J. A network of immediate early gene products propagates subtle differences in mitogen-activated protein kinase signal amplitude and duration. Mol. Cell. Biol. 2004; 24:144-153. [PubMed: 14673150]

2. Murphy LO, Blenis J. MAPK signal specificity: the right place at the right time. Trends Biochem. Sci. 2006; 31:268-275. [PubMed: 16603362] 
3. Palmero I, Serrano M. Induction of senescence by oncogenic Ras. Methods Enzymol. 2001; 333:247-256. [PubMed: 11400340]

4. Ballif BA, Blenis J. Molecular mechanisms mediating mammalian mitogen-activated protein kinase (MAPK) kinase (MEK)-MAPK cell survival signals. Cell Growth Differ. 2001; 12:397-408. [PubMed: 11504705]

5. Karnoub AE, Weinberg RA. Ras oncogenes: split personalities. Nat. Rev. Mol. Cell. Biol. 2008; 9:517-531. [PubMed: 18568040]

6. Karreth FA, Tuveson DA. Modelling oncogenic Ras/Raf signalling in the mouse. Curr. Opin. Genet. Dev. 2009; 19:4-11. [PubMed: 19201597]

7. Lau KS, Haigis KM. Non-redundancy within the RAS oncogene family: insights into mutational disparities in cancer. Mol. Cells. 2009; 28:315-320. [PubMed: 19812895]

8. Schubbert S, Shannon K, Bollag G. Hyperactive Ras in developmental disorders and cancer. Nat. Rev. Cancer. 2007; 7:295-308. [PubMed: 17384584]

9. Chen PC, Wakimoto H, Conner D, Araki T, Yuan T, Roberts A, Seidman CE, Bronson R, Neel BG, Seidman JG, Kucherlapati R. Activation of multiple signaling pathways causes developmental defects in mice with a Noonan syndrome-associated Sos1 mutation. J. Clin. Invest. 2010; 120:43534365. [PubMed: 21041952]

10. Aoki Y, Niihori T, Narumi Y, Kure S, Matsubara Y. The RAS/MAPK syndromes: novel roles of the RAS pathway in human genetic disorders. Hum. Mutat. 2008; 29:992-1006. [PubMed: 18470943]

11. Dougherty MK, Muller J, Ritt DA, Zhou M, Zhou XZ, Copeland TD, Conrads TP, Veenstra TD, Lu KP, Morrison DK. Regulation of Raf-1 by direct feedback phosphorylation. Mol. Cell. 2005; 17:215-224. [PubMed: 15664191]

12. Ritt DA, Monson DM, Specht SI, Morrison DK. Impact of feedback phosphorylation and Raf heterodimerization on normal and mutant B-Raf signaling. Mol. Cell. Biol. 2010; 30:806-819. [PubMed: 19933846]

13. Muller J, Ory S, Copeland T, Piwnica-Worms H, Morrison DK. C-TAK1 regulates Ras signaling by phosphorylating the MAPK scaffold, KSR1. Mol. Cell. 2001; 8:983-993. [PubMed: 11741534]

14. McKay MM, Ritt DA, Morrison DK. Signaling dynamics of the KSR1 scaffold complex. Proc. Natl. Acad. Sci. USA. 2009; 106:11022-11027. [PubMed: 19541618]

15. Corbalan-Garcia S, Yang SS, Degenhardt KR, Bar-Sagi D. Identification of the mitogen-activated protein kinase phosphorylation sites on human Sos1 that regulate interaction with Grb2. Mol. Cell. Biol. 1996; 16:5674-5682. [PubMed: 8816480]

16. Fritsche-Guenther R, Witzel F, Sieber A, Herr R, Schmidt N, Braun S, Brummer T, Sers C, Bluthgen N. Strong negative feedback from Erk to Raf confers robustness to MAPK signalling. Mol. Syst. Biol. 2011; 7:489. [PubMed: 21613978]

17. Sapkota GP, Cummings L, Newell FS, Armstrong C, Bain J, Frodin M, Grauert M, Hoffmann M, Schnapp G, Steegmaier M, Cohen P, Alessi DR. BI-D1870 is a specific inhibitor of the p90 RSK (ribosomal S6 kinase) isoforms in vitro and in vivo. Biochem. J. 2007; 401:29-38. [PubMed: 17040210]

18. Dufresne SD, Bjorbaek C, El-Haschimi K, Zhao Y, Aschenbach WG, Moller DE, Goodyear LJ. Altered extracellular signal-regulated kinase signaling and glycogen metabolism in skeletal muscle from p90 ribosomal S6 kinase 2 knockout mice. Mol. Cell. Biol. 2001; 21:81-87. [PubMed: 11113183]

19. Schneider A, Mehmood T, Pannetier S, Hanauer A. Altered ERK/MAPK signaling in the hippocampus of the mrsk2_KO mouse model of Coffin-Lowry syndrome. J. Neurochem. 2011; 119:447-459. [PubMed: 21838783]

20. Douville E, Downward J. EGF induced SOS phosphorylation in PC12 cells involves P90 RSK-2. Oncogene. 1997; 15:373-383. [PubMed: 9242373]

21. Yaffe MB, Rittinger K, Volinia S, Caron PR, Aitken A, Leffers H, Gamblin SJ, Smerdon SJ, Cantley LC. The structural basis for 14-3-3:phosphopeptide binding specificity. Cell. 1997; 91:961-971. [PubMed: 9428519]

22. Shimamura A, Ballif BA, Richards SA, Blenis J. Rsk1 mediates a MEK-MAP kinase cell survival signal. Curr. Biol. 2000; 10:127-135. [PubMed: 10679322] 
23. Roux PP, Richards SA, Blenis J. Phosphorylation of p90 ribosomal S6 kinase (RSK) regulates extracellular signal-regulated kinase docking and RSK activity. Mol. Cell. Biol. 2003; 23:47964804. [PubMed: 12832467]

24. Chou MM, Blenis J. The $70 \mathrm{kDa}$ S6 kinase complexes with and is activated by the Rho family G proteins Cdc42 and Rac1. Cell. 1996; 85:573-583. [PubMed: 8653792]

25. Fisher TL, Blenis J. Evidence for two catalytically active kinase domains in pp90rsk. Mol. Cell. Biol. 1996; 16:1212-1219. [PubMed: 8622665]

26. Anjum R, Roux PP, Ballif BA, Gygi SP, Blenis J. The tumor suppressor DAP kinase is a target of RSK-mediated survival signaling. Curr. Biol. 2005; 15:1762-1767. [PubMed: 16213824]

27. Ballif BA, Cao Z, Schwartz D, Carraway KL 3rd, Gygi SP. Identification of 14-3-3epsilon substrates from embryonic murine brain. J. Proteome Res. 2006; 5:2372-2379. [PubMed: 16944949]

28. Kettenbach AN, Rush J, Gerber SA. Absolute quantification of protein and post-translational modification abundance with stable isotope-labeled synthetic peptides. Nat. Protoc. 2011; 6:175186. [PubMed: 21293459]

29. Ballif BA, Carey GR, Sunyaev SR, Gygi SP. Large-scale identification and evolution indexing of tyrosine phosphorylation sites from murine brain. J. Proteome Res. 2008; 7:311-318. [PubMed: 18034455]

30. Ballif BA, Roux PP, Gerber SA, MacKeigan JP, Blenis J, Gygi SP. Quantitative phosphorylation profiling of the ERK/p90 ribosomal S6 kinase-signaling cassette and its targets, the tuberous sclerosis tumor suppressors. Proc. Natl. Acad. Sci. USA. 2005; 102:667-672. [PubMed: 15647351]

31. Lechtreck KF, Johnson EC, Sakai T, Cochran D, Ballif BA, Rush J, Pazour GJ, Ikebe M, Witman GB. The Chlamydomonas reinhardtii BBSome is an IFT cargo required for export of specific signaling proteins from flagella. J. Cell Biol. 2009; 187:1117-1132. [PubMed: 20038682]

32. Leighton IA, Dalby KN, Caudwell FB, Cohen PT, Cohen P. Comparison of the specificities of p70 S6 kinase and MAPKAP kinase-1 identifies a relatively specific substrate for p70 S6 kinase: the $\mathrm{N}$-terminal kinase domain of MAPKAP kinase-1 is essential for peptide phosphorylation. FEBS Lett. 1995; 375:289-293. [PubMed: 7498520]

33. Cargnello M, Roux PP. Activation and function of the MAPKs and their substrates, the MAPKactivated protein kinases. Microbiol. Mol. Biol. Rev. 2011; 75:50-83. [PubMed: 21372320]

34. Carriere A, Ray H, Blenis J, Roux PP. The RSK factors of activating the Ras/MAPK signaling cascade. Front. Biosci. 2008; 13:4258-4275. [PubMed: 18508509]

35. Roux PP, Ballif BA, Anjum R, Gygi SP, Blenis J. Tumor-promoting phorbol esters and activated Ras inactivate the tuberous sclerosis tumor suppressor complex via p90 ribosomal S6 kinase. Proc. Natl. Acad. Sci. USA. 2004; 101:13489-13494. [PubMed: 15342917]

36. Crooks GE, Hon G, Chandonia JM, Brenner SE. WebLogo: a sequence logo generator. Genome Res. 2004; 14:1188-1190. [PubMed: 15173120]

37. Bridges D, Moorhead GB. 14-3-3 proteins: a number of functions for a numbered protein. Sci. STKE. 20052005 re 10.

38. Zhang L, Wang H, Liu D, Liddington R, Fu H. Raf-1 kinase and exoenzyme S interact with 14-3-3zeta through a common site involving lysine 49. J. Biol. Chem. 1997; 272:13717-13724. [PubMed: 9153224]

39. Zimmermann S, Moelling K. Phosphorylation and regulation of Raf by Akt (protein kinase B). Science. 1999; 286:1741-1744. [PubMed: 10576742]

40. Corbit KC, Trakul N, Eves EM, Diaz B, Marshall M, Rosner MR. Activation of Raf-1 signaling by protein kinase $\mathrm{C}$ through a mechanism involving Raf kinase inhibitory protein. J. Biol. Chem. 2003; 278:13061-13068. [PubMed: 12551925]

41. Romeo Y, Roux PP. Paving the way for targeting RSK in cancer. Expert Opin. Ther. Targets. 2010; 15:5-9. [PubMed: 20958120]

42. Old WM, Shabb JB, Houel S, Wang H, Couts KL, Yen CY, Litman ES, Croy CH, Meyer-Arendt K, Miranda JG, Brown RA, Witze ES, Schweppe RE, Resing KA, Ahn NG. Functional proteomics identifies targets of phosphorylation by B-Raf signaling in melanoma. Mol. Cell. 2009; 34:115131. [PubMed: 19362540] 
43. McClatchey AI. Neurofibromatosis. Annu. Rev. Pathol. 2007; 2:191-216. [PubMed: 18039098]

44. Bignone PA, Lee KY, Liu Y, Emilion G, Finch J, Soosay AE, Charnock FM, Beck S, Dunham I, Mungall AJ, Ganesan TS. RPS6KA2, a putative tumour suppressor gene at 6q27 in sporadic epithelial ovarian cancer. Oncogene. 2007; 26:683-700. [PubMed: 16878154]

45. Lopez-Vicente L, Armengol G, Pons B, Coch L, Argelaguet E, Lleonart M, Hernandez-Losa J, de Torres I, Ramon y Cajal S. Regulation of replicative and stress-induced senescence by RSK4, which is down-regulated in human tumors. Clin. Cancer Res. 2009; 15:4546-4553. [PubMed: 19584160]

46. Mackeigan JP, Murphy LO, Dimitri CA, Blenis J. Graded mitogen-activated protein kinase activity precedes switch-like c-Fos induction in mammalian cells. Mol. Cell. Biol. 2005; 25:4676-4682. [PubMed: 15899869]

47. Sturm OE, Orton R, Grindlay J, Birtwistle M, Vyshemirsky V, Gilbert D, Calder M, Pitt A, Kholodenko B, Kolch W. The mammalian MAPK/ERK pathway exhibits properties of a negative feedback amplifier. Sci. Signal. 2010; 3 ra90.

48. Fritsche-Guenther R, Witzel F, Sieber A, Herr R, Schmidt N, Braun S, Brummer T, Sers C, Bluthgen N. Strong negative feedback from Erk to Raf confers robustness to MAPK signalling. Mol. Syst. Biol. 7:489. [PubMed: 21613978]

49. Obenauer JC, Cantley LC, Yaffe MB. Scansite 2.0: Proteome-wide prediction of cell signaling interactions using short sequence motifs. Nucleic Acids Res. 2003; 31:3635-3641. [PubMed: 12824383] 


\section{Synopsis}

The extent and duration of Mitogen Activated Protein Kinase (MAPK) signaling govern a diversity of normal and aberrant cellular outcomes. Genetic and pharmacological disruption of the MAPK-activated kinase RSK leads to elevated MAPK activity indicative of a RSK-dependent negative feedback loop. Using biochemical, pharmacological and quantitative mass spectrometry approaches we show that RSK phosphorylates the Ras activator Son of Sevenless homolog 1 (SOS1) in cultured cells on two carboxyl-terminal residues, Ser1134 and Ser1161. Furthermore, we find that RSKdependent SOS1 phosphorylation creates 14-3-3 binding sites. We show that mutating Ser1134 and Ser1161 disrupts 14-3-3 binding and modestly increases and extends MAPK activation. Together these data suggest that one mechanism whereby RSK negatively regulates MAPK activation is via site-specific SOS1 phosphorylation. 
A.

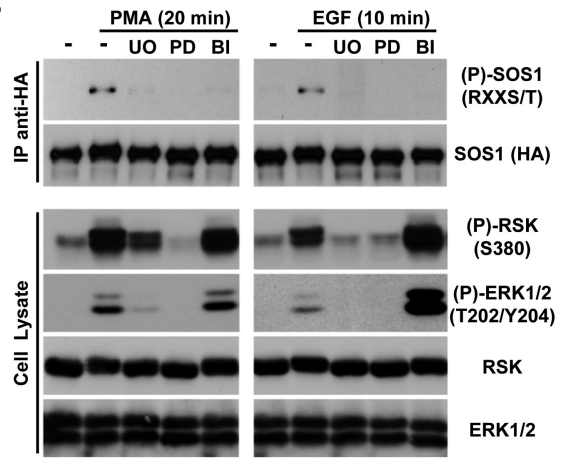

B.

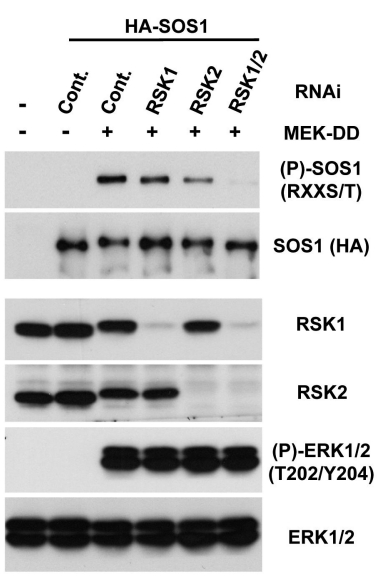

FIGURE 1.

Identification of one or more potential RSK phosphorylation sites in SOS1 using an antiRXXpS-specific motif antibody. (A) PMA and EGF induce phosphorylation of an RXXS motif in SOS1. HEK 293 cells transfected with HA-SOS1 were starved of serum and left untreated or stimulated with PMA or EGF for the indicated times. Twenty minutes prior to stimulation the indicated cultures were treated with U0126 (U0), PD184352 (PD), or BID1870 (BI). HA-SOS1 was immunoprecipitated from whole cell extracts and immune complexes and whole cells extracts were subjected to immunoblotting with the indicated antibodies. (B) RSK1 and RSK2 contribute to MEK-Dependent phosphorylation of SOS1. Cells were treated as in A except with the co-transfection of RNAi and an activated MEK1 allele (MEK-DD) as indicated. 
A.

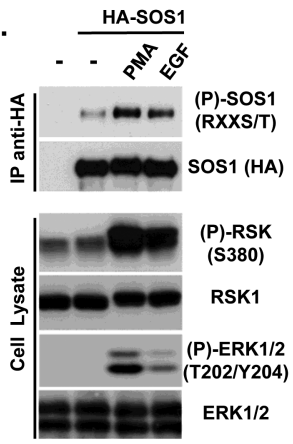

B.

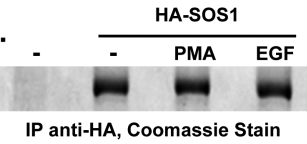

D.

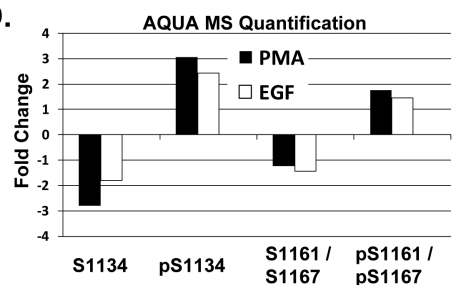

E.

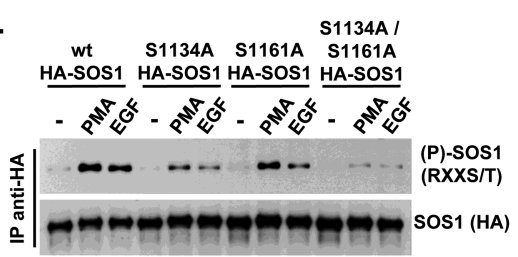

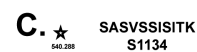
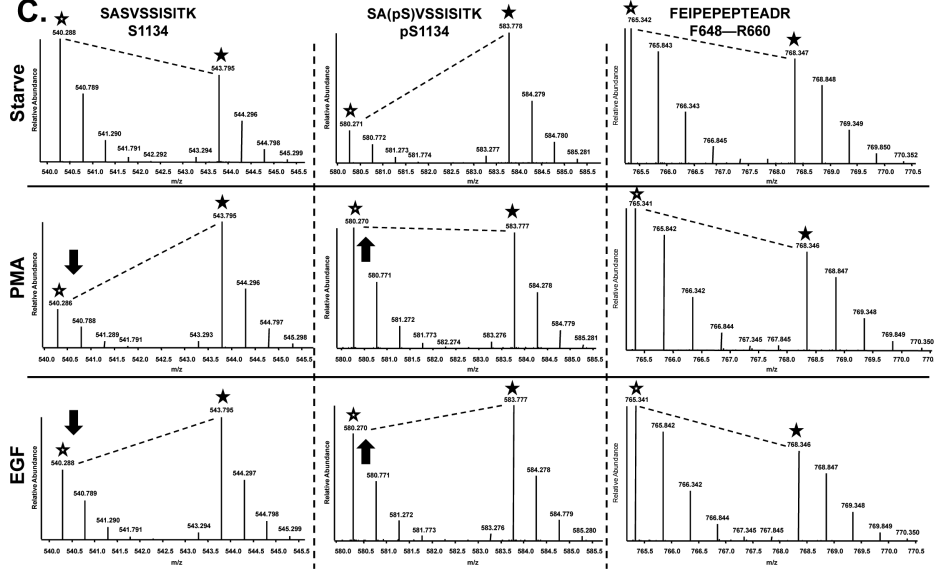

FIGURE 2.

Ser1134 and Ser1161 are the PMA- and EGF-induced SOS1 phosphorylation sites that conform to the minimal RSK consensus motif. (A) HEK 293 cells were treated as described in Figure 1 and whole cell extracts as well as ten percent of each immune complex was subjected to Immunoblotting with the indicated antibodies. (B) $90 \%$ of the immune complexes shown in A were subjected to SDS-PAGE and Coomassie blue staining. (C) Gel bands from B were subjected to in-gel tryptic digestion and extracted peptides were mixed with stable isotope-containing reference peptides. Peptides were subjected to technical replicate LC-MS/MS analyses. Representative example of raw data showing the isotopic envelopes from the MS1 full scans of the stable isotope-containing and native peptides inclusive of Ser1134, phosphoSer1134 and Phe648-Arg660 from starved, PMA-stimulated and EGF-stimulated cells are shown. Monoisotopic peak height for the native (open stars) and labelled (filled stars) peptides are indicated. Dashed lines connect monoisotopic peaks of peptide pairs. Increases and decreases in abundance are indicated by arrows. (D) Averages and standard deviations of technical replicates for fold changes relative to unstimulated are indicated. MS/MS spectra for labelled and native peptides are shown in Supplementary Figure S1. (E) Ser1134 and then Ser1161 are the major RXXS phosphorylation sites in SOS1 following stimulation with PMA and EGF. HEK 293 cells transfected with wild-type HA-SOS1 or the indicated HA-SOS1 mutants were starved of 
serum and stimulated with the indicated factors. Cell extracts were subjected to anti-HA immunoprecipitation and immunoblotting with the indicated antibodies. 
A.

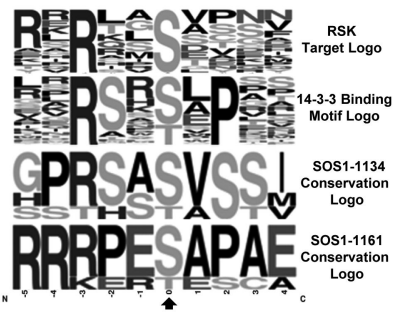

C.

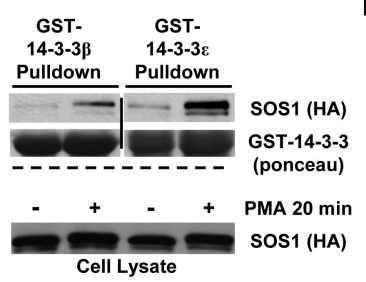

E.

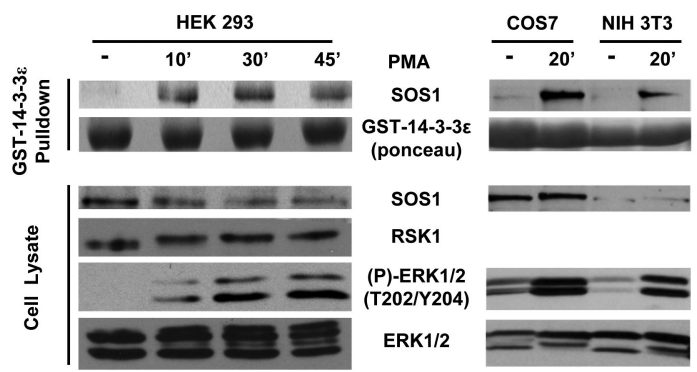

B.

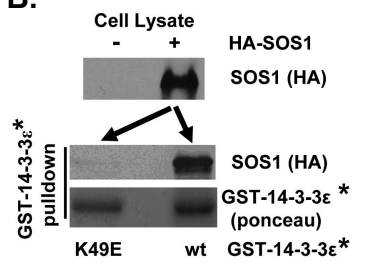

D.

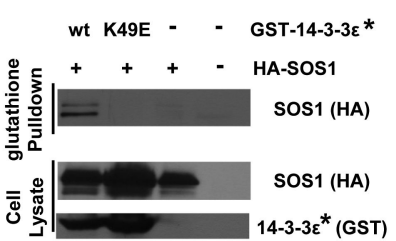

FIGURE 3.

SOS1 binds to 14-3-3 in a stimulus and likely phospho-dependent manner. (A) Comparison of Weblogo frequency plots of the RSK target motif (upper panel), the top 500 Scansite [49] predicted 14-3-3 binding motifs in proteins from the Swiss Prot database, and the evolutionary conservation around SOS1 Ser1134 and Ser1161 (lower two panels) from sequences presented in Supplementary Figure S2. (B) SOS1 binds to wild-type but not Lys49Glu 14-3-3e in pulldown assays. HEK 293 cells were mock transfected or transfected with HA-SOS1. Whole cell extracts were subjected to immunoblotting as indicated. Equal portions of cell extract containing HA-SOS1 were subjected to pulldown assays using bacterially-produced GST-14-3-3e wild-type (wt) or Lys49Glu as indicated. Pulldowns were subjected to immunoblotting with anti-HA antibodies. Amounts of each GST-14-3-3e fusion protein are shown by Ponceau staining of the membrane prior to immunoblotting. "**" refers to wild-type or mutant GST-14-3-3 $\varepsilon$ as indicated. (C) PMA-stimulated SOS1 interacts with GST-14-3-3 $\beta$ and $\varepsilon$. Pulldowns and immunoblots were conducted with the indicated fusion proteins and antibodies as described in B above. (D) SOS1 binds to co-transfected GST-14-3-3e wild-type but not GST-14-3-3e Lys49Glu. HEK 293 cells growing in complete media were mock transfected or transfected with HA-SOS1 with or without the indicated expression plasmids for GST-14-3-3e variants. Cell extracts (lower panel) and glutathione agarose pulldown assays were subjected to immunoblotting with the indicated antibodies (upper panel). (E) Endogenous SOS1 binds to GST-14-3-3e following stimulation with PMA. HEK 293, COS7 and NIH3T3 cells were starved of serum for 16 hours following stimulation with PMA for the indicated times. Cell extracts (lower panel) and GST-14-3-3e pulldown assays (upper panel) were subjected to immunoblotting with the indicated 
antibodies. Amounts of GST-14-3-3e fusion protein are shown by Ponceau staining of the membrane prior to immunoblotting. 
A.

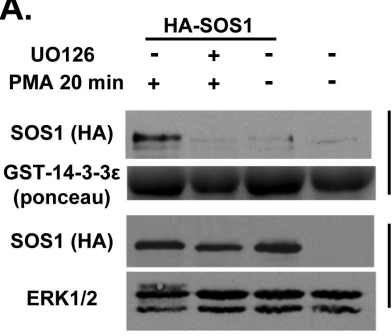

B.



C.

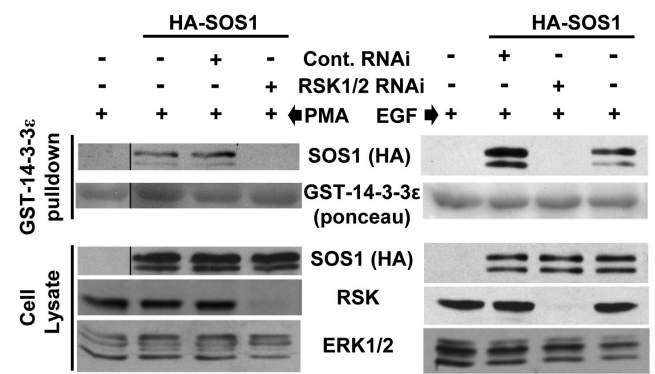

D.

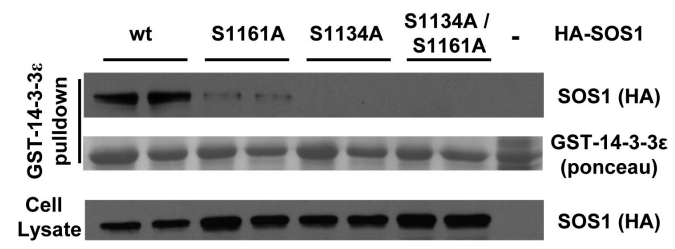

FIGURE 4.

RSK phosphorylates SOS1 inducing 14-3-3 binding. (A) Pharmacological disruption of MEK1/2 activation blocks PMA-induced SOS1 binding to 14-3-3e. HEK 293 cells mocktransfected or transfected with HA-SOS1 were starved for 16 hours. Where indicated, cells were pre-treated with U0126 for 30 minutes prior to stimulation with PMA for 20 minutes. Cell extracts and GST-14-3-3e pulldown assays were subjected to immunoblotting with the indicated antibodies. Amounts of GST-14-3-3e fusion protein are shown by Ponceau staining of the membrane prior to immunoblotting. (B) Constitutively active, myristoylated (Myr)-RSK1 induces SOS1 binding to $14-3-3 \varepsilon$ in the absence of stimulation. Cells were treated as in A except for the co-transfection of Myr-RSK1 (avian) as indicated. "Av" indicates an antibody specific to avian RSK1. Starved cells were lysed and extracts were subjected to pulldown assays and immunoblotting as in A. (C) RSK is required for PMA and EGF-induced binding of 14-3-3e to SOS1. Cells were treated as in A except for the cotransfection of RNAi for RSK1/2 or a control RNAi where indicated. Starved cells were then treated with either PMA (20') or EGF (10') prior to pulldowns and immunoblotting. (D) Phosphorylation at Ser1134 and Ser1161 mediate the binding of SOS1 to 14-3-3e. HEK 293 cells were transfected with the indicated HA-SOS1 wild-type (wt) or serine-to-alanine mutant constructs. Cell extracts were subjected to GST-14-3-3e pulldown assays and immunoblotting as in A. 


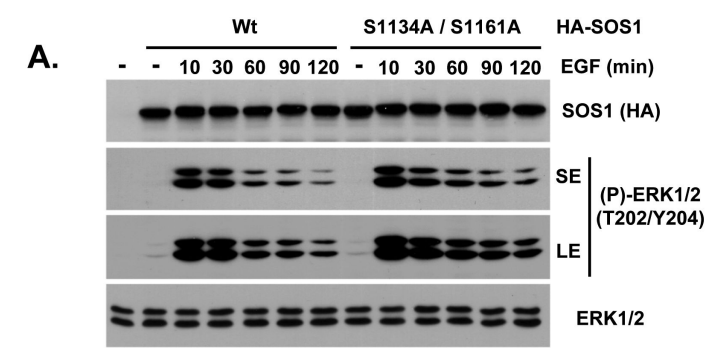

B.


C.

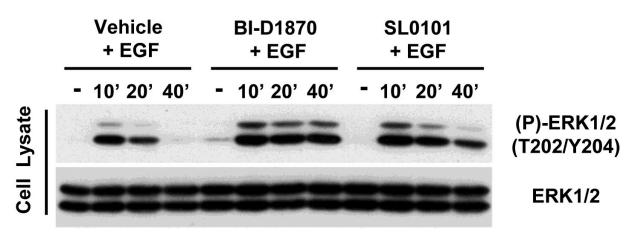

FIGURE 5.

RSK phosphorylation of SOS1 negatively regulates ERK1/2 phosphorylation. (A) SOS1 Ser1134Ala/Ser1161Ala increases the magnitude and duration of EGF-stimulated ERK1/2 phosphorylation. The indicated HA-SOS1 wild-type and mutant constructs were transfected into HEK 293 cells. Cells were starved and stimulated with EGF for the indicated times prior to lysis. Cell extracts were subjected to SDS-PAGE and immunoblotting with the indicated antibodies. Both a short exposure (SE) and a long exposure (LE) are provided for phospho-ERK1/2 specific blots. (B) The left panel shows relative phospho-ERK1/2 quantified by densitometry (as described in the methods) from three experiments similar to the one shown in A. The right panel shows the mean fold increase in ERK1/2 phosphorylation of the three experiments comparing wild-type (HA-SOS1) and Ser1134Ala-, Ser1161Ala HA-SOS1. Error bars represent the standard error of the mean. One-tailed, unequal variance T-test p-values are indicated for a comparison of the means of the wild-type and mutant (S2A) phospho-ERK1/2 levels. Stars indicate significance at a 95\% confidence interval. (C) As shown for HEK 293 cells in Fig.1A, inhibition of RSK reveals the presence of a negative feedback loop. Mouse embryonic fibroblasts were starved of serum and then stimulated with EGF or pre-treated with RSK inhibitors BI-D1870 or SL-0101for 30 minutes prior to stimulation with EGF, and immunoblotting of extracts as indicated. 


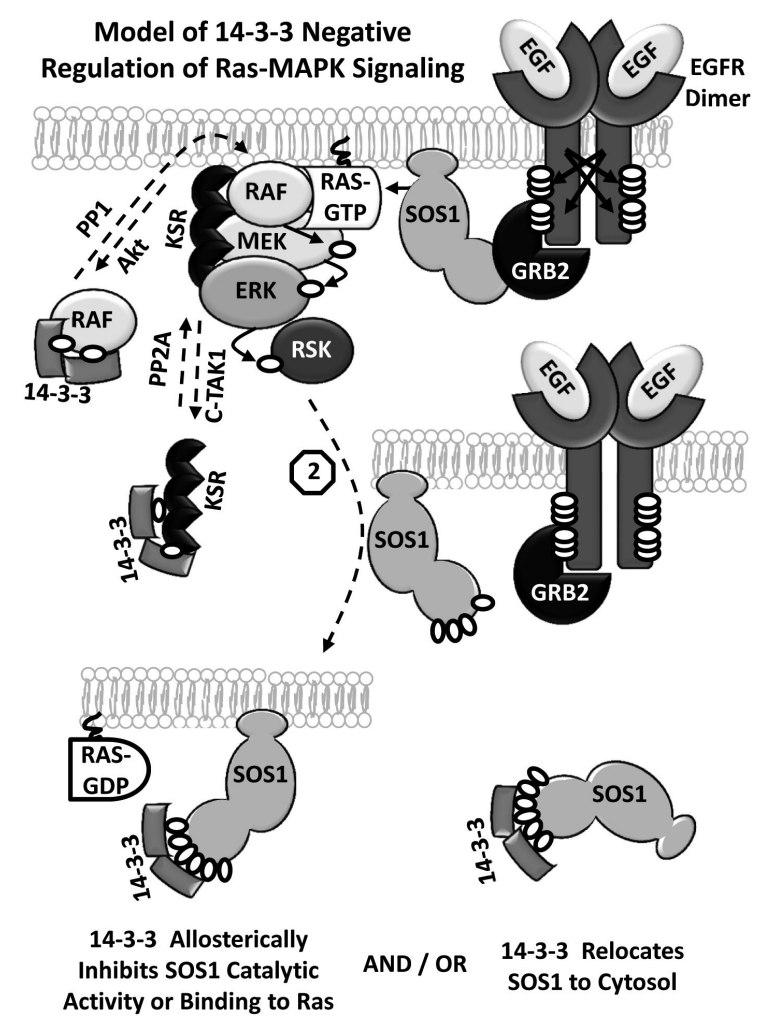

FIGURE 6.

Model of a two stage phospho-dependent Ras-MAPK feedback loop to negatively regulate SOS1. Activation of Ras-MAPK signaling by EGF engaged with EGFR induces autophosphorylation of EGFR and the recruitment of Grb2 bound to SOS1. SOS1, which also interacts with lipids at the plasma membrane, activates Ras which can then interact with Raf leading to sequential activation of MEK and ERK. KSR localizes the three kinases as a cassette at the plasma membrane. 14-3-3 proteins prevent membrane localization of both KSR and RAF unless dephosphorylated by PP2A and PP1 respectively. ERK activation leads to the activation of RSK. In stage one of the negative feedback loop on SOS1, ERK phosphorylates several sites in the C-terminus of SOS1 which reduces its binding to Grb2. This may lead to SOS1 diffusing away from the proximity of the activated signaling complex. In stage two RSK phosphorylates SOS1 at Ser1134 and Ser1161 leading to 14-3-3 binding which might reduce SOS1 catalytic activity or prevent its interaction with Ras. Alternatively, 14-3-3 might reduce the capacity for SOS1 to bind to the plasma membrane and thereby attenuate Ras-MAPK signaling. 